\section{Creationin silicon}

John L. Casti

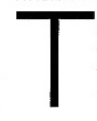

he idea of creating a mechanical intelligence that can display human-like thought processes has been a dream pursued by the artificial-intelligence (AI) community from the dawning of the computer age. But since no one really knows how humans think, the test of whether a machine intelligence has the 'right stuff' has been based on the behaviouristic test, originally proposed by Alan Turing ${ }^{1}$ in 1950 , that the machine is intelligent - human-style - if a human interrogator can't tell the difference between interacting with a machine and with another human. Now a computer program put together by William McCune (Argonne National Lab., Illinois, USA) has found a solution ${ }^{2}$ to a major mathematical problem that could be said to easily pass a Turing test for mathematical creativity, giving ammunition to supporters of the 'strong-AI' thesis that there is no intrinsic barrier to a computer thinking just like you and me.

The problem solved by McCune's program involves a conjecture in boolean algebras that a certain set of three equations constitutes a basis for such an algebra. The problem was first posed by Herbert Robbins in the 1930s, and was worked on by a number of mathematicians, including the famed logician Alfred Tarski. McCune's automated reasoning program, which is a general-purpose 'prover' of the truth or falsity of logical expressions, was given a statement of the Robbins problem, along with a few simple parameters to restrict the search, none of which was particularly focused on boolean algebras.

The program searched an infinite space of logical expressions and found a proof of the conjecture. In fact, the program found several different proofs of the conjecture, depending on various parameters, especially the maximum length of any expression encountered in the search process. The first proof of the Robbins conjecture took a total of about eight days of searching using three workstations.

Of course, computers have been used many times in the past to resolve mathematical conjectures. Probably the most wellknown of these efforts was the work by Appel and Haken in 1976 to prove the famed four-colour conjecture ${ }^{3}$ (that four colours are enough to fill in any planar map, without having adjacent regions of the same colour). A similar exhaustive computer search by Clement Lam in 1988 showed the nonexistence of projective planes of order 10 . But in these cases the mathematicians outlined the proof beforehand, then wrote special-purpose software to check the large, but finite, number of cases needed to establish the theorem. So there was a pretty good idea before the computation started as to how long it would take, and that an answer would be found.

In contrast, in his computer proof of the Robbins conjecture, McCune had no expectations of any sort that a proof would emerge when he started his program working on the problem. Moreover, what finally emerged was an explicit proof that can be checked by hand and by independent proof-checking programs. This contrasts strongly with the Appel-Haken and Lam computer proofs, whose correctness depends on the correctness of the software.

The solution of the Robbins conjecture was found by a program designed to reason, not to solve a specific problem. Whether this constitutes a 'creative' act or not is open to debate. But as Larry Wos, the supervisor of the computer reasoning project at Argonne says, "It's a sign of power, of reasoning power... We've taken a quantum leap forward". Wos predicts that this result may mark the beginning of a new era in mathematics, one in which mathematicians focus on producing interesting conjectures, leaving their proof or disproof to computer programs. These sorts of proofs by machine suggest that perhaps there is a finer line than one might have thought between mechanical search and creative acts.

But even if this line of work on automated theorem-proving does revolutionize mathematical practice, what does it mean for creative thought in general? Can we justify extrapolating a creative proof of a mathematical conjecture like the Robbins Conjecture into a belief that machines can construct equally creative works of art in other areas like poetry, literature or music? Or will they be confined to human-like acts of inspiration only in areas, like mathematics or chess, that are, by and large, outside the realm of everyday human experience and emotion? For years the strong-AI community has argued that there is no significant difference between the two. Anti-AI crusaders, such as John Searle of Berkeley, have stated that the gap is unbridgeable. McCune's work at least suggests that the whole issue may soon move from the realm of philosophical speculation to that of laboratory experiment.

John L. Casti is at the Santa Fe Institute, Santa Fe, New Mexico 87501, USA.

\footnotetext{
1. Turing, A. M. Mind 59, 434-460 (1950).

2. McCune, W. J. Automated Reasoning (in the press).

3. Appel, K. \& Haken, W. Bull. Am. Math. Soc. 82, 711-712
} (1976).
Daedalus

Celestial torque

Conventional astronomy studies the stars and planets by their radiation alone. The gravitational fields of the planets extend to the Earth, but are ignored. Could a sensitive gravimeter detect them? At first sight, this seems impossible. Like a spacecraft, the Earth is in free fall among the planets. The gravimeter falls with it, and no force can be developed between them. However, free fall only masks the direct field of a heavenly body; its field gradient is still detectable. A satellite in free fall feels a torque from the gradient of the Earth's gravity - its slightly greater intensity on the side nearer the Earth. Indeed, gravity-gradient effects can be used to stabilize a satellite, keeping its long axis pointing at the Earth. Similar effects should be felt on the Earth due to masses in space. Olympic high-jumpers have been advised to wait till the Sun or Moon is directly overhead; its upward pull will gain them a extra fraction of a millimetre.

So Daedalus began to invent an earthbound gravity-gradiometer for detecting masses in space. It was a long rigid rod, freely suspended and equipped with delicate interferometric sensors. Nearby heavenly bodies would impose torques on it. As the Earth rotated, the intensity and angle of the torques would change, revealing the masses and positions of the bodies responsible. But he then realized that nature has already done it. The ocean tides are nothing but a natural gravity-gradient detector on a vast scale. They are, of course, dominated by the Moon and the Sun. Yet the nearer planets must raise tides of their own, typically a few micrometres high.

DREADCO physicists are now designing a tide-gauge to reveal them. A large vertical pipe will be anchored to the side of some atoll in deep mid-ocean. Its bottom will be so deep that wave action will not enter; its surface will respond only to tidal influences. A mirror floating on that surface will form part of a laser interferometer, or even a quantum tunnelling device, revealing the tidal flow with almost atomic precision. Unperturbed by daylight or cloud cover, it will open a new window on the Solar System. Since tidal effects intensify inversely as the cube of the distance, even satellites in low orbit might raise detectable tides. And should there be any of the proposed astronomical 'dark matter' in our neighbourhood, or some black asteroid on a collision course with Earth, it might elude our telescopes, but would show up unfailingly on the interferometric tide-gauge.

David Jones 